\title{
BENEFÍCIOS DOS EXERCÍCIOS RESPIRATÓRIOS NO YOGA EM MULHERES ADULTAS NA FAIXA ETÁRIA DE 40 A 90 ANOS
}

Evanize Kelli Siviero Romarco

Claudia Bolsonaro Ferreira Lima

\section{Resumo}

Este trabalho teve por objetivo verificar os benefícios dos exercícios respiratórios na prática do Yoga e sua influência sobre tensões, dores musculares e ansiedade-traço. Para pesquisa foram avaliadas doze mulheres adultas na faixa etária de 40 a 90 anos, divididas em três grupos aleatoriamente, sendo $\mathrm{G}(1)$ realizou atividades do Yoga e exercícios respiratórios, grupo $\mathrm{G}(2)$ realizou as mesmas atividades do Yoga sem os exercícios respiratórios e grupo $\mathrm{G}(\mathrm{C})$ não realizou atividade alguma, mantendo-se como grupo controle. Para a coleta de dados, foi aplicado o questionário diagnóstico ás participante no início e no termino do quarto mês e um teste de ansiedade. Os resultados finais indicaram melhora significativa nos pontos de dores e tensões musculares, nos grupos $\mathrm{G}(1)$ e $\mathrm{G}(2)$ como esperado na literatura pesquisada. Quanto à ansiedade-traço, não ocorreram mudanças significativas em nenhum dos três grupos.

\section{Palavras-Chave}

Yoga. Exercícios respiratórios; Ansiedade-traço; Dores e tensões musculares.

\section{BENEFITS OF RESPIRATORY EXERCISES IN YOGA IN ADULT WOMEN BETWEEN 40 TO 90 YEARS OF AGE}

Evanize Kelli Siviero Romarco

Claudia Bolsonaro Ferreira Lima

\begin{abstract}
This work is aimed at verifying the benefits of breathing exercises in the practice of Yoga and their effect on stress, anxiety and muscle pain. The aim of this work was to verify the Group G (1) did some yoga and breathing exercises, Group G (2) performed the same activities but the breathing exercises and Group G (C) did not do any activity and was considered control group. To collect data, a diagnostic questionnaire and an anxiety test were used in the beginning of the program and in the end of the fourth month. The final results showed significant improvement on muscle pain and tension in groups G (1) and G (2) as expected on the researched literature. As for anxiety, no significant changes occurred in any of the three groups.
\end{abstract}

\section{Key-Words}

Yoga; Breathing exercises; Anxiety-dash; Pain and muscle tension. 


\section{INTRODUÇÃO}

O Yoga e a respiração estão intrinsecamente relacionados. Os antigos mestres do Yoga já sabiam disso, assim começaram a testar e analisar formas diferentes de respirações, seus efeitos e seus benefícios, desenvolvendo dessa forma, exercícios respiratórios designados pranãyamas (ALLEGRO, 2004).

Gharote (2002) escreve que os diferentes tipos de respirações no Yoga são necessários e desejáveis, para que a circulação pulmonar melhore. Sua pesquisa mostra que durante o pranãyama não se obtém mais quantidade de ar na inspiração, e sim absorve mais oxigênio deste ar inspirado quando comparado a respiração espontânea. Isso se deve ao controle consciente do ato respiratório, onde o diafragma, também controlado, acarreta um efeito positivo sobre órgãos abdominais (rins, intestino, pâncreas e fígado), ativando os chamados "centros de energia" (gânglios ou núcleos nervosos do corpo) gerando, assim, uma melhora qualitativa na fisiologia respiratória e circulatória, colaborando com a nutrição de órgãos vitais.

De acordo com Pinto (2006) ao realizar as asãnas ${ }^{1}$ e os pranãyamas com regularidade, consegue-se desenvolver respostas benéficas ao corpo como diminuição de adrenalina, tranqüilização da mente, melhora da força, equilíbrio, firmeza, flexibilidade e harmonia, todos esses fatores poderão exercer grande influência nas atividades e reações diárias na vida de seus praticantes.

Portanto, por acreditar na importância dos exercícios respiratórios desenvolvidos na prática do Yoga resolveu-se investigar seus benefícios, principalmente no que diz respeito ao grau de ansiedade-traço, dores e tensões musculares, pesquisando e aplicando-os em mulheres adultas na faixa etária de 40 a 90 anos.

\section{YOGA E RESPIRAÇÃO}

"Respiração é vida e vida é respiração" (GHAROTE, 2002, p. 47) assim o autor descreve a importância que se dá à respiração, pois segundo ele, raras vezes nos conscientizamos desse processo, a menos que haja algum incômodo, quando há_disfunções no sistema respiratório ou alto grau de ansiedade, ou nos deixando sem ar e angustiados.

O professor de Yoga e biólogo Anderson Allegro (2004) escreve que os antigos mestres do Yoga sabiam da importância da respiração na nossa vida através das observações e experiências que realizavam, por

\footnotetext{
${ }^{1}$ Asãnas: posturas psicofísicas ou atitudes corporais (ALLEGRO, 2004). 
isso começaram a testar e analisar diferentes tipos de exercícios de respiração, desenvolvendo o que denominaram pranãyamas ${ }^{2}$.

Segundo a filosofia do Yoga, ao respirar oxigênio, também absorve-se prâna, que é “[...] a alma da Força a Força da Energia, o Princípio Universal, a Essência de todo movimento, desenvolvimento e realização" (GARCIA, [197-.], p.3) é entendido segundo essa filosofia, como a força, a vitalidade e energia que se manifesta em todas as formas de vida. Através da respiração do Yoga, é possível perceber de acordo com Silva (2002) nossos batimentos cardíacos, nosso corpo, nossas sensações, aumentado assim a consciência corporal do praticante.

Com do aprendizado das práticas respiratórias do Yoga, ocorre uma conscientização da forma correta de se respirar, aprende-se a controlar a fase de inspiração e de expiração, a realizar pausas respiratórias exigindo da mente uma participação ativa no aparelho respiratório. Participação voluntária, que segundo Guyton (1992) se processa no córtex e em outros centros superiores e vão até os neurônios espinais estimulando os músculos respiratórios. Através do controle voluntário da respiração, ocorre no organismo mecanismo que regulam a respiração e o sistema cardiovascular, de maneira involuntária, interagindo o sistema nervoso simpático e parassimpático

Quando o indivíduo pratica pranãyamas, consegue estimular o sistema nervoso autônomo a ponto de inibir o sistema simpático, ocorrendo então maior estímulo do sistema nervoso parassimpático, o que diminui consequentemente a freqüência cardíaca e relaxa os músculos esqueléticos. Com a prática assídua dos pranãyamas os receptores químicos localizados no centro respiratório, sensíveis à elevação do gás carbônico arterial, respondem menos a concentração elevada de CO2 na fase expiratória, aumentando conscientemente essa fase espontânea da respiração e produz "[...] um treinamento tão forte do sistema nervoso autônomo que há um aumento das variações de freqüência cardíaca mesmo quando a pessoa não está praticando[...]”(SANTAELLA; DI BENEDETTO, 2006, p. 93).

Segundo os autores citados acima, esse fator é muito importante, pois quanto_menor a freqüência cardíaca, menor débito cardíaco e, por conseguinte menor a variação da pressão arterial, visto que, duas equações explicam essa afirmativa: PA= DCxRV (PA - pressão arterial; DC_débito cardíaco e RV-

\footnotetext{
${ }^{2}$ Pranãyamas: palavra de origem sânscrita do radical prana que significa alento ou energia vital e yama que significa domínio, portanto pranãyamas é o domínio da energia existente no ar e no Universo, a qual inalamos durante a prática desses exercícios respiratórios (ALLEGRO, 2004)
} 
resistência vascular periférica) e $\mathrm{DC}=\mathrm{VsxFC}(\mathrm{DC}$ - débito cardíaco; Vs - volume sistólico; FCfreqüência cardíaca) diminuindo a probabilidade de o praticante ter problemas cardíacos.

Ao realizar os exercícios respiratórios do Yoga, inicia um controle dos músculos que fazem os movimentos respiratórios (musculatura voluntária), o que não acontece na respiração voluntária e na intensa $^{3}$, ou seja, criam-se espaços internos a partir da expansão torácica e o ar penetra nos pulmões no ritmo e quantidade determinado pelo praticante.

Sabe-se que a respiração tem dupla função: a de alimentar através da absorção do oxigênio todas as células corporais e a de limpar, pela eliminação do dióxido de carbono os resíduos corporais que não necessários ao organismo - processo denominado hematose. E, como o oxigênio é imprescindível à vida, quando um indivíduo tem a respiração inadequada ou mesmo deficiente, ocorre muitas vezes, a redução da vitalidade, da capacidade de movimentação muscular e articular, levando-o a se sentir cansado facilmente, à depressão, à exaustão, à ansiedade, prejudicando muitas vezes sua auto-expressão (SAMPAIO, 2005).

Com a prática consciente dos exercícios do Yoga, consegue-se inverter esse processo, como mostra alguns estudos já citados. A melhora na qualidade de vida é aparente, ao passo que a saúde se restabelece.

\section{A ANSIEDADE}

Quando um indivíduo se encontra inseguro, por uma expectativa de algum perigo ou ameaça eminente, com medo de perder algo real ou imaginário e quando sente ansiedade/angústia que é permanente e que pode desencadear uma psicopatologia, entende-se que isto é ansiedade (COZZANI. et al., 1997).

Também, a ansiedade pode ser considerada como um estado emocional com componentes psicológicos e fisiológicos, mas que faz parte das experiências humanas sendo propulsora muitas vezes, do desempenho, não trazendo qualquer prejuízo, mas quando se torna desproporcional à situação ou quando não existe algo real que a desencadeia, torna-se patológica (ANDRADE; GORENSTEIN, 2006).

Se ocorrer com muita freqüência e sem causa real saindo do limite considerado suportável fisicamente, pode originar descargas automáticas de nossos neurônios sobre os órgãos e glândulas por eles enervados,

\footnotetext{
${ }^{3}$ A respiração normal e tranqüila é realizada pelo movimento do diafragma, quase que inteiramente. Já durante a respiração intensa, os músculos abdominais se contraem forçando o conteúdo abdominal para cima, contra a parte inferior do diafragma, ajudando na expiração (SANTAELLA \& DI BENEDETTO, 2006)
} 
acarretando aumento dos ácidos e enzimas alterando, assim, nosso metabolismo, interferindo na respiração, aumentando a sudorese e taquicardia, deixando o indivíduo apreensivo (COZZANI. et al., 1997), podendo também aumentar seu gasto energético.

Siviero (2001/2002) relata que quando a pessoa se encontra apreensiva, nervosa e preocupada, pode acarretar grande ativação do seu sistema nervoso autônomo, o que poderá se transformar em emoções que fogem ao controle, provocando doenças somáticas, angústias, tristeza, dores múltiplas, estresse, ansiedade, tensões musculares, e outras patologias.

Muitas dessas doenças somáticas podem ser tratadas através dos exercícios de respiração do Yoga, como relata o psiquiatra Rennó Jr. (2006) da Universidade de São Paulo. Segundo ele, a respiração ofegante e acelerada, típica das pessoas diagnosticadas com ansiedade, aumenta o tônus muscular estimulando o sistema nervoso a reagir de uma forma a lutar ou fugir. O psiquiatra relata que essa reação deixa o organismo pronto para reagir de forma extrema frente a qualquer tipo de problema, levando o indivíduo a se tornar agressivo. Isso acaba afetando, também, a regeneração celular proporcionando o que ele chama de meia força, ou seja, com pouca energia corpórea no seu dia-a-dia.

Para melhor compreensão e avaliação da ansiedade, Spielberg (1970) apud Cozzani, (1997) adotou duas formas distintas e complementares para avaliação da ansiedade: ansiedade-traço e ansiedade-estado.

Ansiedade-traço: entendida como aquela permanente da personalidade do indivíduo, ocorre de formas diferentes e individuais, determinando como o indivíduo reage frente a situações que ele entende como ameaçadoras

Ansiedade-estado: entendida como estado emocional transitório, caracterizado por sentimentos desagradáveis como tensão e apreensão que são percebidos pelo aumento da atividade do sistema nervoso autônomo (ANDRADE; GORENSTEIN, 2006).

Relacionando os exercícios do Yoga como descritos na literatura pesquisada com estados de ansiedade, entende-se que com a prática contínua e bem orientada dos mesmos, podem ocorrer benefícios quanto ao alívio de dores e tensões musculares, bem como no grau de ansiedade, pois Gulmini (2006) relata que quando modificamos nossas atitudes diárias, quando aprimoramos nossa capacidade de observação, 
melhoramos o equilibro de nossas necessidades individuais, ao passo que percebemos melhor nossas reações e nossas dores e angústias.

\section{MÉTODO}

Este trabalho é de natureza descritiva e exploratória, sendo o mesmo uma pesquisa de campo do tipo participante. Aplicou-se o Questionário Diagnóstico elaborado por uma das pesquisadoras, que continha questões sobre pontos de dores e tensões musculares. Para medir o grau de ansiedade utilizou-se o questionário IDATE II, sendo os dois questionários aplicados no início e final da pesquisa.

Doze mulheres adultas na faixa etária 40 a 90 anos foram às participantes, sendo as mesmas indicadas por um médico ortopedista da cidade de Barretos- SP, o mesmo foi procurado por uma das pesquisadoras para que indicasse algumas pacientes que ele entendia necessitavam realizar as atividades do Yoga, sendo também informado quanto ao teor da pesquisa. As participantes foram divididas aleatoriamente em três grupos: Yoga com exercícios respiratórios - grupo (G1); Yoga sem exercícios respiratórios - grupo (G2) e um terceiro grupo que não realizou qualquer atividade - grupo (GC). Os dados foram tratados de forma quanti-qualitativa, pois este método segundo Karim (2004) constitui-se de uma maneira que possibilita comparar resultados quantitativos por meio de controles estatísticos.

\section{ANÁLISE E DISCUSSÃO DOS DADOS}

Quanto ás mudanças que ocorreram em relação à intensidade das tensões e dores musculares, pode-se observar no Gráfico um, que o grupo G (1) teve alterações quanto à diminuição dos pontos de dores e tensões musculares comparando-se o pré com o pós-teste. Também foi medido o tempo de prática das participantes, para verificar a influência dessa variável. 


\section{Gráfico 1- Grupo G1 - mudanças em relação às tensões e dores musculares}



Entende-se matematicamente ao se comparar o pré-teste com o pós-teste do Grupo G(1), que a Participante um teve uma diminuição de $50 \%$ de suas dores e tensões musculares, enquanto que a sua freqüência nas aulas foi de 48\%; a Participante dois não apresentou mudanças significativas nesse quesito permanecendo como observado no gráfico com os mesmos pontos de dores e tensões, a sua freqüência nas aulas foi de 36\%; já a Participante três teve uma diminuição de $84,61 \%$ e sua freqüência nas aulas foi de 52\% e a Participante quatro obteve uma mudança de 33,33\%, quanto à diminuição de dores e tensões musculares e sua freqüência nas aulas foi de $52 \%$.

Quanto às mudanças ocorridas em três participantes deste grupo, podemos dialogar com o que Ferreira (2005) descreve quanto à respiração reeducativa ser uma maneira de facilitar o relaxamento e alongamento dos músculos responsáveis pelo processo respiratórios, bem como proporciona liberação de tensões musculares aumentando a liberdade articular do organismo de forma geral. Ou seja, quando realiza as respirações adequadas durante a prática do Yoga, mais controle há do sistema nervoso autônomo e, por conseguinte, maior relaxamento e menor contração muscular com menos dores.

$\mathrm{Na}$ análise desse gráfico pode ser colocada também como destaque a assiduidade das participantes, representada pela Participante dois em contra posição as Participantes um, três e quatro, observando-se que a única participante que não apresentou melhoras no quesito dores e tensões musculares, foi a que menos freqüentou as aulas durante o período de coleta. Filla (2006) relata que o Yoga deve ser realizado num processo contínuo, respeitando-se os limites e necessidades individuais para que os resultados 
apareçam, pode-se entender então que a assiduidade às aulas permitiu a continuidade do trabalho bem como os resultados observados neste grupo.

Relativo ao Gráfico dois, comparando-se o pré-teste com o pós-teste, nota-se que este Grupo, que realizou as posturas do Yoga sem os exercícios respiratórios adequados, as Participantes um e dois tiveram ausência de dores e tensões musculares, e a freqüência nas aulas foi de $44,44 \%$ respectivamente; já na Participante três percebe-se um aumento de $34 \%$ de suas dores e tensões musculares, enquanto que a participação nas aulas desta foi de $77,77 \%$.

\section{Gráfico 2- Grupo G2- mudanças em relação às tensões e dores musculares pré e pós teste.}



Quanto aos resultados do Grupo G (2), podemos relacionar as mudanças ocorridas nas participantes um e dois recorrendo à literatura pesquisada, entendendo que na prática do Yoga, executa-se movimentos articulares, musculares e de relaxamento, conseguindo-se efeitos no sistema músculo-esquelético, possibilitando um melhor alongamento e relaxamento muscular. Mesmo sem os exercícios adequados de respiração, ocorreram em duas das três participantes, mudanças no quesito analisado. Ou seja, os benefícios que os exercícios do Yoga apresentaram neste grupo, independeram dos exercícios de respiração.

Já a Participante três de acordo com a análise do Gráfico 2, não obteve diminuição nos pontos de dores e tensões e sim, um aumento destes, sendo esta a de maior freqüência às aulas. Pode-se então, relacionar o aumento de pontos de dores e tensões musculares ao analisarmos o resultado obtido no Questionário 
IDATE II, pois esta participante apresentou, comparando o pré-teste com o pós-teste, grau de ansiedade considerado elevado.

Considerando-se que, a participante três, ao realizar os exercícios de Yoga com o grau de ansiedade elevado e sem os exercícios de respiração adequados, não conseguiu desenvolver com propriedade as posturas trabalhadas no Yoga e consequentemente suas tensões musculares não diminuíram, dificultando o que esta prática ensina quanto à questão de que quanto maior conforto, menos tensão teremos, ocorrendo menor ativação neuromuscular e por conseqüência, menos fibras contraídas e menos dores.

Quanto às participantes do Grupo Controle - $G(C)$ também podemos verificar no Gráfico três as mudanças ocorridas, relativas às dores e tensões musculares, durante os quatro meses de prática.

\section{Gráfico 3- Grupo Controle: G C}

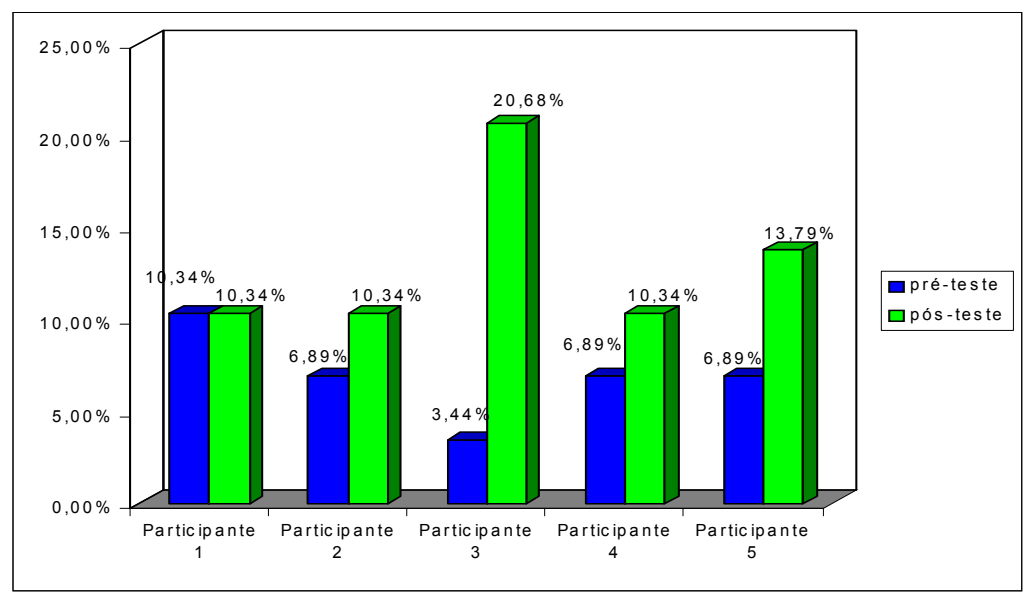

Portanto, pode-se verificar que no Grupo G(1) três das quatro participantes, obtiveram alterações relativas à diminuição de dores e tensões musculares após prática do Yoga com as posturas (asãnas), os exercícios respiratórios (pranãyamas) e o relaxamento (nidra) compatível com dados da literatura encontrados; no Grupo $\boldsymbol{G ( 2 )}$ que realizou somente as posturas e o relaxamento, duas das três participantes, obtiveram melhora nos pontos de dores e tensões musculares, e somente uma teve esses pontos aumentados, já no Grupo $\boldsymbol{G}(\boldsymbol{C})$ quatro das cinco participantes tiveram seus pontos de dores e tensões musculares aumentados e apenas uma manteve os mesmos pontos. 
$\mathrm{Na}$ análise dos gráficos apresentados quanto à mudança em relação às tensões e dores musculares das participantes e compatível com a literatura, observa-se que o Yoga com exercícios respiratórios sendo realizado com freqüência e correta orientação melhorou a qualidade de vida da Participante três $(52 \%$

freqüente nas aulas) em contra posição a Participante dois, que não apresentou mudança alguma e foi observada um freqüência nas aulas de $36 \%$, inferior as outras participantes.

Com relação à ansiendade-traço utilizou-se neste estudo para quantificá-lo o IDATE II, realizando a somatória dos valores dado a cada afirmação, variação 0 a 4 pontos, para estabelecer próprio escore. $\mathrm{O}$ valor final demonstrou a pontuação obtida por cada participante. Para determinar o grau de ansiedadetraço compararmos com a tabela de escore pré-estabelecida por Spilberger apud Fioravanti (2006). As indicações para a classificação entre os escores são: grau baixo: de 0 a 30; grau mediano: de 31 a 49; grau elevado: igual ou maior que 50.

Ao ser analisado o IDATE II, verificou-se que no grupo $\boldsymbol{G}$ (1) os escores ficaram entre 46 a 49 na primeira coleta, significando grau mediano de ansiedade, já na segunda coleta a participante um apresentou grau elevado de ansiedade, as participantes dois, três e quatro apresentaram grau mediano de ansiedade tanto na primeira quanto na segunda coleta; no grupo $\boldsymbol{G ( 2 )}$ os escores ficaram entre 35 a 49 relativos às três participantes na primeira coleta, significando grau mediano de ansiedade, na segunda coleta somente a participante três apresentou grau elevado de ansiedade (escore 51), as outras duas participantes não tiveram alterações; no grupo $\boldsymbol{G}(\boldsymbol{C})$ as cinco participantes apresentaram grau mediano de ansiedade na primeira coleta, os escores ficaram entre 40 a 48, já na segunda coleta somente a participante dois apresentou grau elevado de ansiedade (escore 50), as outras quatro participantes não tiveram alterações quanto ao grau de ansiedade.

Ao se analisar o Questionário IDATE II, observou-se que não ocorreram alterações significativas, em relação à ansiedade-traço, nos três grupos analisados. Considerando-se que a ansiedade-traço está atrelada a vários fatores como, por exemplo, a expectativa de algum perigo, a tensão e a apreensão, entende-se que para os benefícios em relação a está característica de personalidade sejam obtidos, é necessário uma intervenção mais específica.

Para próximas discussões, poderemos utilizar outras áreas da filosofia Yoga para avaliar os parâmetros da ansiedade. Será que a pratica do Yoga com todo estudo da filosofia altera a personalidade do individuo? 


\section{CONSIDERAÇÕES FINAIS}

Após os quatro meses de pesquisa, houve através de gráficos a confirmação da literatura, com objetivos parciais alcançados Quando esta pesquisa foi iniciada, acreditávamos que o Yoga era uma maneira de

interferir beneficamente na vida dos praticantes desta atividade, principalmente nos seus pontos de dores e tensões musculares e na ansiedade-traço.

Durante a interferência das aulas de Yoga, nos dois grupos $G(1)$ e $G(2)$ observamos que a maioria teve melhoras nos pontos de tensões e dores musculares, já no grupo controle $G(C)$ que não realizou atividade alguma, ocorreram na grande maioria das participantes, alterações relativas ao aumento desses pontos, comprovando que o Yoga contribuiu para mudanças nos grau de tensão e dores musculares de seus praticantes, de acordo com a literatura pesquisada.

Enfim, podemos considerar que o Yoga é uma prática que mesmo que seus elementos (asãnas, pranãyamas e nidra) sejam realizados separadamente, traz benefícios para o indivíduo que o pratica. Segundo Rodrigues (2006) estes benéficos aparecem como resposta natural para as necessidades de uma época cheia de tensões e pressa, como a que atualmente temos vivenciado.

Quanto à ansiedade-traço, não ocorreram alterações nos escores em relação à diminuição, sendo necessária talvez, uma análise mais detalhada deste elemento e até mesmo a ampliação do tempo de prática. Como escreve Rodrigues (2006) o Yoga foi elaborado para se desenvolver, preservar e restabelecer nossa saúde, como também para integrar todas as funções do ser humano, mas cada indivíduo precisa dirigir a prática para o que mais necessita ou deseja.

Percebe-se assim, nos grupos estudados por quatro meses, que o Yoga auxilia na qualidade de vida dos seus praticantes disciplinados, refletindo numa melhora fisiológica comprovada.

\section{REFERÊNCIAS}

ALLEGRO, A. Respirações. Disponível em: http//www.yogasite.com.br>. Acesso em: 06 dez 2004. ANDRADE, L. H. S. G.; GORENSTEIN, C. Aspectos gerias das escalas de avaliação de ansiedade. Disponível em: $<$ http//www.hcnet.usp.br $>$. Acesso em: 04 jun. 2006. 
COZZANI, M. et.al. Ansiedade: interferências no contexto esportivo. In: MACHADO, A. A. (Org.). Psicologia do esporte: Temas emergentes. Jundiaí: Ápice: 1997.

FERREIRA, J. E. R. A importância do processo respiratório nas aulas de Educação Física e práticas esportivas. Disponível em: <http//www.efdesportes.com.br>. Acesso em: 09 mai. 2005.

FILLA, J. A. M. Yoga-Nidra: a ciência do relaxamento. In: RODRIGUES, R. M. (Org.). Estudos sobre o yoga. São Paulo: Phorte, 2006. p. 61-66.

FIORAVANTI, A. Ansiedade. Disponível em: <http//www.fotolog.terra.com.br $>$. Acesso em: 04 out. 2006.

GARCIA, S. G. Curso fundamental de hatha-yoga. São Paulo: Iracema, s/d? [197-]

GHAROTE, M. L. Yoga Aplicada: da teoria à prática. In: ROJO, M. (Adaptação Técnica). São Paulo: Phorte, 2002.

GULMINI, L. C. As várias histórias do Yoga. In: RODRIGUES, M. R. (Org.). Estudos sobre o yoga. São Paulo: Phorte, 2006.

GUYTON, A. C. Tratado de fisiologia médica. 8. ed. Rio de Janeiro: Guanabara Koogan, 1992.

KARIM, T. M. Planejamento de auto-avaliação. Universidade do Estado de Mato Grosso. Disponível em: $<$ http//www.unemat.br/avaliação $>$. Acesso em: 04 out. 2004.

PINTO, G. S. Yoga. Disponível em: <http//www.25horas.com.br>. Acesso em: 11 mar. 2006.

RENNÓ J. R. J. Ioga \& medicina. Época. n. 425, p. 66-74, Jul. 2006. p. 66-74.

RODRIGUES, M. R. Yoga aplicada: da teoria à prática. 2. ed. São Paulo: Phorte, 2006.

SAMPAIO. S. A. B. Respiração e Tvp. Disponível em: <http//www.abep-tvp.com/artigos/> Acesso em: 06 jun. 2005.

SANTAELLA; DI BENEDETTO. Considerações fisiológicas. In: RODRIGUES, R. M. (Orgs.). Estudos sobre o Yoga. São Paulo: Phorte, 2006.p.75-95.

SILVA, T. G. O Yoga e o equilibrio do ser humano. 2002. 42f. Dissertação (mestrado em Educação Física) - Instituto de Biociências, Universidade Estadual Paulista "Julio de Mesquita Filho, Rio Claro, 2002.

SIVIERO, E. K. A influência dos exercícios suaves sobre a relação estados emocionais e tensão muscular. Revista Hispeci \& Lema, Bebedouro: v. 6, p. 59-66. 2001/02. 


\section{Evanize Kelli Siviero Romarco}

Universidade Federal de Viçosa/UFV

\section{Claudia Bolsonaro Ferreira Lima}

Faculdades Integradas Fafibe

\section{Referência do Artigo}

\section{ABNT}

ROMARCO, E. K. S., LIMA, C. B. F. Benefícios dos exercícios respiratórios no yoga em mulheres adultas na faixa etária de 40 a 90 anos. Conexões, v. 6, n. 3, p. 78-90, 2008.

\section{APA}

Romarco, E. K. S., \& Lima, C. B. F. (2008). Benefícios dos exercícios respiratórios no yoga em mulheres adultas na faixa etária de 40 a 90 anos. Conexões, 6 (3), 78-90.

\section{VANCOUVER}

Romarco EKS, Lima CBF. Benefícios dos exercícios respiratórios no yoga em mulheres adultas na faixa etária de 40 a 90 anos. Conexões, 2008; 6(3), 78-90.

\section{Recebido em 25/10/08}

Aceito 18/11/08 